\title{
Burns and Fire Prevention
}

\section{A SAFER CANDLE PROJECT - SOUTH AFRICA}

doi:10.1136/injuryprev-2012-040590a.1

PP Mtambeka, D Schulman, N du Toit, H Rode, AB Van As. Childsafe South Africa

Red Cross War Memorial Children's Hospital

Background There are two main causes of shack fires in South Africa: Fallen candles and paraffin-related burns. These fires lead to devastating consequences and huge economic losses.

Aim/Goal The goal of this project is to facilitate and promote safer use of candles in a glass jar, with the ultimate aim to prevent fires, burn injuries and deaths caused by fallen candles, targeting individuals and families who live in informal homes.

Material and Methods Childsafe South Africa personnel conducted a number of trials to test the "Candle in a glass jar" concept. In 2006, the project was piloted in an informal area that exclusively uses candles as source of light. Further demonstration and educational sessions have taken place and over 100,000 jar units have been distributed to various communities to date.

Results The Safer Candle Project is six years old, and has become one of Childsafe's established programmes. It has been widely accepted and been approved by World Wide Fund for Nature SA (WWF) for Earth Hour 2011\& 2012.

Significance The idea of candle in the glass jar is recommended as its very simple and preventative measure to reduce fires mainly caused by candles tipping. One of its advantages is that it places no financial burden on families, as there are virtually no costs involved.

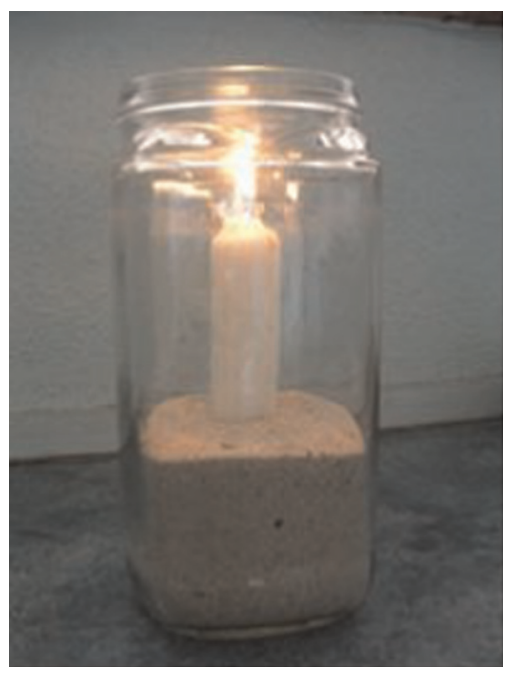

